\title{
Oxidative stress and metabolic markers in pre- and postnatal polycystic ovary syndrome rat protocols
}

This article was published in the following Dove Press journal: Journal of Inflammation Research

\section{Lady Serrano Mujica' \\ Alessandra Bridi' ${ }^{1,2}$ \\ Ricardo Della Méa' \\ Vitor Braga Rissi' \\ Naiara Guarda ${ }^{3}$ \\ Rafael Noal Moresco ${ }^{3}$ \\ Melissa Orlandin Premaor ${ }^{4}$ \\ Alfredo Quites Antoniazzi' \\ Paulo Bayard Dias \\ Gonçalves' \\ Fabio Vasconcellos \\ Comim ${ }^{1,4}$}

'Laboratory of Biotechnology and Animal Reproduction, BioRep, Federal University of Santa Maria, Santa Maria, Rio Grande do Sul, ${ }^{2}$ Department of Veterinary Medicine, University of São Paulo, São Paulo, ${ }^{3}$ Laboratory of Clinical Biochemistry, Department of Clinical and Toxicological Analysis, ${ }^{4}$ Department of Clinical Medicine, Federal University of Santa Maria, Santa Maria, Rio Grande do Sul, Brazil

Correspondence: Fabio Vasconcellos Comim

Department of Clinical Medicine, Federal University of Santa Maria, Av Roraima 1000, Building 26, Room 1337, Santa Maria, Rio Grande do Sul 97105900, Brazil

Tel +55 5532208752

Email fabio.comim@ufsm.br
Background: Several studies have described an enhanced inflammatory status and oxidative stress balance disruption in women with polycystic ovary syndrome (PCOS). However, there is scarce information about redox markers in the blood of androgenized animal models. Here, we evaluated the serum/plasma oxidative stress marker and metabolic parameter characteristics of prenatal (PreN) and postnatal (PostN) androgenized rat models of PCOS.

Materials and methods: For PreN androgenization ( $\mathrm{n}=8), 2.5 \mathrm{mg}$ of testosterone propionate was subcutaneously administered to dams at embryonic days 16, 17, and 18, whereas PostN androgenization $(\mathrm{n}=7)$ was accomplished by subcutaneously injecting $1.25 \mathrm{mg}$ of testosterone propionate to animals at PostN day 5. A unique control group $(n=8)$ was constituted for comparison. Results: Our results indicate that PostN group rats exhibited particular modifications in the oxidative stress marker, an increased plasma ferric-reducing ability of plasma, and an increased antioxidant capacity reflected by higher albumin serum levels. Post $\mathrm{N}$ animals also presented increased total cholesterol and triglyceride-glucose levels, suggesting severe metabolic disarrangement.

Conclusion: Study findings indicate that changes in oxidative stress could be promoted by testosterone propionate exposure after birth, which is likely associated with anovulation and/ or lipid disarrangement.

Keywords: animal models of PCOS, oxidative stress, prenatal, postnatal

\section{Introduction}

Polycystic ovary syndrome (PCOS) is a complex endocrine and metabolic disorder impacting $5 \%-10 \%$ of women at reproductive age. ${ }^{1-3}$ Although PCOS has been identified mainly by reproductive features (oligo-amenorrhea, hyperandrogenism, polycystic ovary appearance), other metabolic and inflammatory conditions, including disruption in cholesterol and glucose levels and the oxidative stress balance, have also been reported. ${ }^{4-10}$ A recent publication has found that total oxidative stress and antioxidant capacity were increased in PCOS against controls. ${ }^{11}$ In this study, which also evaluated the four phenotypes of PCOS, a higher oxidative stress was related to increased androgens, plasma glucose, and triglycerides, and decreased apo $\mathrm{A}_{1}$ concentrations. ${ }^{11}$

Studies in rodent models replicate many of the abnormalities observed in PCOS women and, for this reason, have been used to explore the pathophysiological basis of the disorder. ${ }^{12-17}$ Currently, there is scarce information about the redox state in the blood of PCOS rats, once the majority of the studies have focused most of their attention on the ovaries and liver, fat, and muscle tissues..$^{17,29,35}$

Therefore, the aim of this study was to compare the oxidative stress profile in two different phenotypes obtained by prenatal (PreN) and postnatal (PostN) androgenization 
protocols (both with testosterone propionate). This research worked with an anestrous rat model of PCOS (PostN androgenized rat or PostN group), an estrous rat model (PreN androgenized rat or PreN group), and an androgenized PostN estrous rat model (PostN L group). ${ }^{18,19}$

Our results indicate that modifications in the oxidative stress markers in the blood occurred in the presence of severe reproductive and metabolic disarrangements observed in the female rats submitted to PostN androgenization with testosterone propionate.

\section{Methods}

\section{Animals}

This study was approved by the Ethics Committee on Animal Use (CEUA) of the Federal University of Santa Maria (UFSM), Brazil, under protocol number 100/14. The procedures with animals were in agreement with the guidelines of the Brazilian National Council of Control of Animal Experimentation that follows the "Principles of Laboratory Animal Care" established by the National Institutes of Health, Bethesda, MD, USA.

Overall, 46 female Wistar rats (Rattus norvegicus var. albinus) were used in this study and housed at the Laboratory of Biotechnology and Animal Reproduction, BioRep, UFSM. The animals were maintained at a temperature of $22^{\circ} \mathrm{C}, 55 \%-65 \%$ humidity under artificial illumination on a light-dark cycle of 12:12 h, with daylight from 7 am to $7 \mathrm{pm}$. Food and water were given ad libitum.

A total of 30 female rats were submitted to the protocol for synchronization of estrus. They received an intraperitoneal injection of $10 \mathrm{IU}$ of equine chorionic gonadotropin (eCG; Folligon ${ }^{\mathrm{TM}}$; Intervet, São Paulo, Brazil), followed $48 \mathrm{~h}$ later by 10 IU of human chorionic gonadotropin (hCG; Pregnyl ${ }^{\text {TM}}$; Organon, Cascavel, Brazil), and were placed with a male for $24 \mathrm{~h}$. Matches were controlled; vaginal plug was checked every $12 \mathrm{~h}$. Observation of the vaginal plug was considered as the first day of pregnancy. Female rat pups were divided into four groups for androgenization by treatment with testosterone propionate or two control groups. Dams were maintained with their pups until weaning (21 days). PreN hormone exposure was accomplished by the treatment of pregnant dams during embryonic days 16,17 , and 18 through a subcutaneous injection of $2.5 \mathrm{mg}$ testosterone propionate (Androgenol ${ }^{\mathrm{TM}}$; Hertape Calier, Juatuba, Brazil) (PreN group), whereas vehicle control exposures were accomplished by similar treatment of pregnant dams with $2.5 \mathrm{mg}$ of corn oil (control PreN). PostN hormone exposures were performed by the treatment of 5-day-old animals through a subcutaneous injection of $1.25 \mathrm{mg}$ testosterone propionate
(PostN group), whereas vehicle control PostN 5-day-old animals received a subcutaneous injection of $1.25 \mathrm{mg}$ of corn oil (control PostN). ${ }^{19}$ The final groups were as follows: PreN ( $n=8)$, PostN ( $n=7)$, and control group (control PreN with control PostN) $(n=8)$. Another androgenized group, PostN leuprolide (PostN L n=7), included the treatment with an intramuscular (im) injection of $0.40 \mathrm{mg}$ of leuprolide acetate depot (Lectrum ${ }^{\mathrm{TM}}$; Sandoz International $\mathrm{GmbH}$, Holzkirchen, Germany) in 2-day-old rats before PostN androgenization with testosterone propionate. Information of other groups of leuprolide treatment including the number of animals per group (eg, PreN androgenized with leuprolide) is available in the Supplementary materials.

\section{Euthanasia and sample collection}

At the age of 110 days, the animals were transferred and then anesthetized with isoflurane by administering tramadol chloride (Tramadol ${ }^{\text {TM}}$; Pfizer, São Paulo, Brazil) intramuscularly (20-40 mg/kg). Between 9 am and 10 am, blood samples were collected before the animals were finally sacrificed using cardiac puncture under deep anesthesia in the absence of pedal and corneal reflexes. Blood samples were centrifuged at $4^{\circ} \mathrm{C}$ and $5000 \mathrm{rpm} / 4696 \times g$ (Sorvall-Thermo Scientific, Asheville, NC, USA) for $15 \mathrm{~min}$ to separate the blood solid components from the serum and plasma (ethylenediaminetetraacetic acid) and stored at $-80^{\circ} \mathrm{C}$.

\section{Laboratory measurements}

Total blood cholesterol, high-density lipoprotein cholesterol (HDL-C), low-density lipoprotein cholesterol (LDL-C) triglyceride, albumin, and glucose were measured enzymatically in serum using a commercial assay kit (LabTest Diagnostics, Lagoa Santa, Brazil).

\section{Ferric-reducing ability of plasma (FRAP)}

FRAP was assessed as previously described. ${ }^{20}$ In brief, the FRAP reagent was freshly prepared and warmed at $37^{\circ} \mathrm{C}$ by mixing the following solutions: 1$) 0.3 \mathrm{M}$ sodium acetate buffer solution ( $\mathrm{pH} 3.6), 2) 10 \mathrm{mM}$ 2,4,6-tripyridyl-1-5-triazine in $40 \mathrm{mM} \mathrm{HCl}$ solution, and 3) $20 \mathrm{mM} \mathrm{FeCl}_{3}$ solution at the ratio of 10:1:1 (v/v/v). Plasma $(10 \mu \mathrm{L})$ was incubated with $90 \mu \mathrm{L}$ of FRAP reagent in a microplate for $30 \mathrm{~min}$ at room temperature in the dark. Subsequently, the level of absorbance of the mixture was measured at the wavelength of $595 \mathrm{~nm}$ using a spectrophotometer. The FRAP values were calculated by using a calibration standard curve of $\mathrm{FeSO}_{4}(0-2000 \mu \mathrm{M})$. All measurements were performed at the same time. The intra-assay coefficient of variation was between $1 \%$ and $2 \%$. 


\section{Measurements of advanced oxidation protein product (AOPP) levels in serum}

Samples were prepared as follows: in a tube, $20 \mu \mathrm{L}$ of serum from each rat was diluted into $100 \mu \mathrm{L}$ in phosphate-buffered saline, followed by the addition of $10 \mu \mathrm{L}$ of $1.16 \mathrm{M} \mathrm{KI}$ and $20 \mu \mathrm{L}$ of absolute acetic acid. The absorbance of the reaction mixture was immediately read using a SpectraMax 1601 spectrophotometer (Molecular Devices, Sunnyvale, CA, USA) at $340 \mathrm{~nm}$ against a blank containing $100 \mu \mathrm{L}$ of phosphate-buffered saline, $20 \mu \mathrm{L}$ of acetic acid, and $10 \mu \mathrm{L}$ of KI solution. ${ }^{21}$ As the linear range of chloramine-T absorbance at $340 \mathrm{~nm}$ is between 0 and $100 \mu \mathrm{M}, \mathrm{AOPP}$ concentrations were expressed in $\mu \mathrm{M}$ chloramine- $\mathrm{T}$ equivalents. All measurements were performed at the same time. The intra-assay coefficient of variation was $4 \%$.

\section{Total oxidation status (TOS)}

TOS of serum was measured using a colorimetric measurement method. ${ }^{22}$ Briefly, $225 \mu \mathrm{L}$ of Reagent 1 (xylenol orange $150 \mu \mathrm{M}, \mathrm{NaCl} 140 \mathrm{mM}$, and glycerol $1.35 \mathrm{M}$ in $25 \mathrm{mM}$ $\mathrm{H}_{2} \mathrm{SO}_{4}$ solution, $\mathrm{pH} 1.75$ ) was mixed with $35 \mu \mathrm{L}$ of serum sample, and the absorbance of each sample was read spectrophotometrically at $560 \mathrm{~nm}$ as a sample blank. Subsequently, $11 \mu \mathrm{L}$ of Reagent 2 (ferrous ion [5 $\mathrm{mM}$ ] and $o$-dianisidine [10 $\mathrm{mM}$ ] in $25 \mathrm{mM} \mathrm{H}_{2} \mathrm{SO}_{4}$ solution) was added to the mixture for $\sim 3-4 \mathrm{~min}$. After mixing, the last absorbance was read at $560 \mathrm{~nm}$. The analytical sensitivity of the method was found to be 0.0076 absorbance/amount $(\mathrm{AX} / \mu \mathrm{M})$. The assay was calibrated with $\mathrm{H}_{2} \mathrm{O}_{2}$, and the results are expressed in terms of micromolar $\mathrm{H}_{2} \mathrm{O}_{2}$ equivalent per liter $\left(\mu \mathrm{mol} \mathrm{H}_{2} \mathrm{O}_{2}\right.$ equiv $/ \mathrm{L}$ ). The detection limit of the method was determined by evaluating the zero calibrator 10 times. All measurements were performed at the same time. The intra-assay coefficient of variation was $6.5 \%$.

\section{Statistical analysis}

The statistical analysis was performed using GraphPad Prism 7.0 (GraphPad Software, Inc., La Jolla, CA, USA). Comparisons among the groups were performed by analysis of variance (ANOVA) followed by post hoc (Tukey) comparison test. In the absence of a normal distribution, verified by Shapiro-Wilk test, the data were analyzed by a Kruskal-Wallis test, followed by Dunn's post hoc test. Proportion among groups was compared by the Fisher's test. Differences between independent variables of two groups were accessed by the Student's $t$-test or Mann-Whitney $U$-test according to the presence or absence of a normal distribution. Significance was assumed at $P<0.05$.

\section{Results \\ Weight of the animals}

PreN androgenized rats showed a lower weight at first PostN day (mean $\pm \mathrm{SD} ; 5.16 \pm 0.21 \mathrm{~g}$ ) in comparison with PostN androgenized (6.56 $\pm 0.4 \mathrm{~g})$ and control $(6.35 \pm 0.44 \mathrm{~g})$ groups $(P<0.001)$ (Figure 1A). At day 60, PreN rats continued to be lighter than PostN and control rats: the mean $\pm \mathrm{SD}$ of weight was 197.3 $\pm 8.0 \mathrm{~g}$ for PreN, 219.5 $\pm 1.5 \mathrm{~g}$ for_PostN, and $210.5 \pm 13.3 \mathrm{~g}$ for control rats $(P=0.01)$ (Figure 1B). Finally, at 110 days, all groups displayed similar weights: the mean \pm SD of $308.8 \pm 15.2$, $314 \pm 15.0$, and $316.5 \pm 10.67 \mathrm{~g}$, respectively (Figure 1C). Groups subjected to leuprolide acetate treatment did not exhibit any modifications in the total weight (data not shown).

\section{Oxidative stress markers and antioxidant capacity (albumin) in serum and plasma}

PostN group rats displayed a significant increase in FRAP, a marker of direct oxidation, compared to PreN group rats. The mean \pm SD of FRAP in PreN was $369.2 \pm 103 \mu \mathrm{mol} / \mathrm{L} / \mathrm{g}$ protein, whereas that in the PostN group was $962 \pm 210.8 \mu \mathrm{mol} / \mathrm{L} / \mathrm{g}$ pro-
A

Day 1

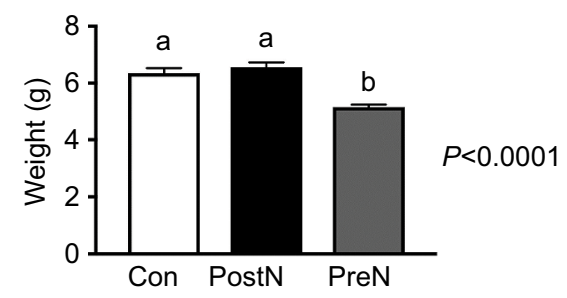

B

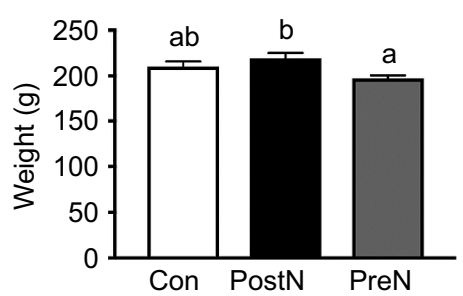

C Day 110

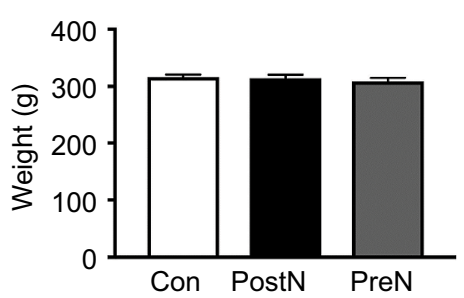

Figure I Weight of rats at days I (A), 60 (B), and I I0 (C) of life.

Notes: Con $(n=8)$; Post $N$ androgenized $(n=7)$; PreN androgenized $(n=8)$. (A) ANOVA $P<0.000$ I; (B) ANOVA $=0.01$. Distinct letters indicate a statistical significant difference (adjusted $P$ level $<0.05$ ) obtained with multi-comparison Tukey's test.

Abbreviations: ANOVA, analysis of variance; Con, controls; PostN, postnatal; PreN, prenatal. 
A

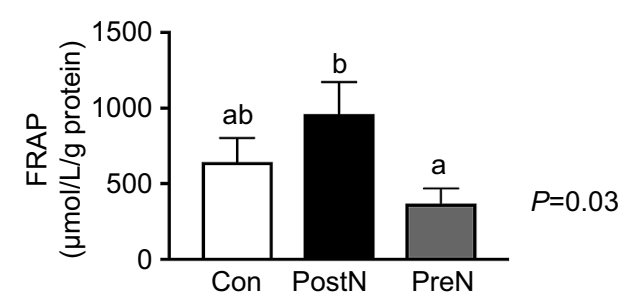

C

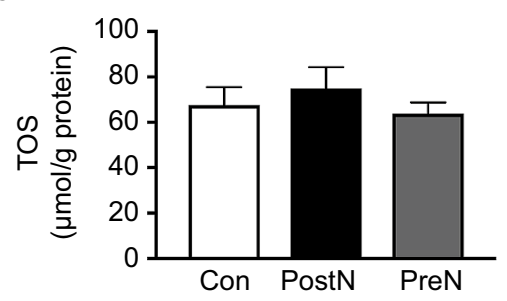

B

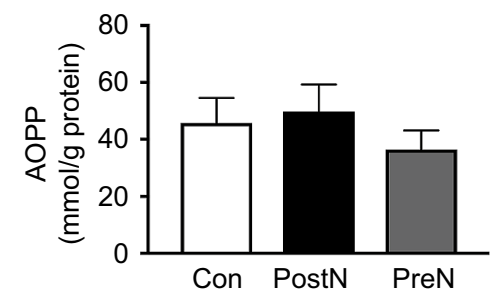

D

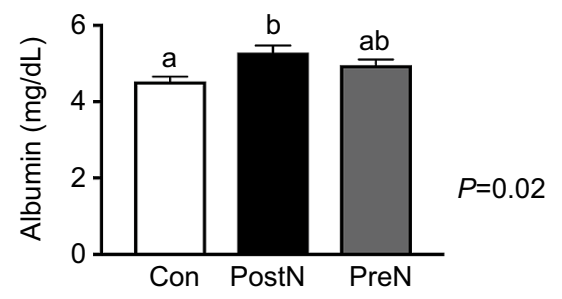

Figure 2 Oxidative stress markers in $\mathrm{C}$, PreN, and Post $\mathrm{N}$ rats.

Notes: Values of oxidants FRAP (A), AOPP (B), TOS (C), and anti-oxidant, albumin (D) in the blood of female rats. Con ( $n=8$ ); PostN androgenized ( $\mathrm{n}=7$ ); PreN androgenized $(n=8)$. (A) ANOVA $P=0.03$; (D) ANOVA $P=0.02$. Distinct letters indicate a statistical significant difference (adjusted $P$ level $<0.05)$ obtained with multicomparison Tukey's test.

Abbreviations: ANOVA, analysis of variance; AOPP, advanced oxidation protein product; Con, controls; FRAP, ferric-reducing ability of plasma; PostN, postnatal; PreN, prenatal; TOS, total oxidation status.

tein $(P=0.03)$ (Figure $2 \mathrm{~A})$. The mean $\pm \mathrm{SD}$ of FRAP in controls was $644.3 \pm 258.6 \mu \mathrm{mol} / \mathrm{L} / \mathrm{g}$ protein. The difference between PostN and controls did not reach statistical significance.

AOPP, another marker of direct oxidative stress, was comparable among the three groups (Figure 2B). The PreN, PostN, and control values were similar, with the mean \pm SD of $36.46 \pm 20.1$, $49.75 \pm 23.32$, and $4585 \pm 213 \mathrm{mmol} / \mathrm{g}$ protein, respectively.

Values for TOS, which estimates the final oxidant status, were superimposed among the three groups. As shown in Figure $2 \mathrm{C}$, similar features were observed in the PreN (mean \pm SD $63.78 \pm 14.68 \mu \mathrm{mol} / \mathrm{L} / \mathrm{g}$ protein), PostN (mean $\pm \mathrm{SD} 74.77 \pm 24.9 \mu \mathrm{mol} / \mathrm{L} / \mathrm{g}$ protein), or control (mean \pm SD $67.5 \pm 20.9 \mu \mathrm{mol} / \mathrm{L} / \mathrm{g}$ protein) groups. Notably, neonatal treatment with leuprolide acetate showed no effect on FRAP, AOPP, or TOS in all groups (Figure S1A-I).

Levels of serum albumin, a surrogate marker of antioxidant capacity, were elevated in the PostN group (mean \pm $\mathrm{SD} 5.28 \pm 0.18 \mathrm{mg} / \mathrm{dL}$ ) versus the control group (mean $\pm \mathrm{SD}$ $4.53 \pm 0.12 \mathrm{mg} / \mathrm{dL})(P=0.01)$. Intermediate values (mean $\pm \mathrm{SD})$ of $4.95 \pm 0.4$ were exhibited in the PreN group (Figure 2D). The treatment with leuprolide in the PostN L group was associated with a significant reduction in albumin levels in comparison with PostN rats (Figure S1K). These changes did not occur with controls (Figure S1J) or with PreN rats (Figure S1L).

\section{Biochemical variables}

Total cholesterol levels were significantly reduced in PreN rats (mean $\pm \mathrm{SD} 77.25 \pm 11.4 \mathrm{mg} / \mathrm{dL}$ ) in comparison with those in PostN rats (mean $\pm \mathrm{SD} 100.7 \pm 11.71 \mathrm{mg} / \mathrm{dL})(P=0.01)$ (Figure 3A). Differences between the total cholesterol levels in the control group (mean $\pm \mathrm{SD} 86.43 \pm 5.25$ ) versus PostN almost reached significance $(P=0.052)$. HDL-C and LDL-C levels were similar between the three groups (Figure $3 \mathrm{~B}$ and C). Notably, the HDL levels decreased in the PreN group after neonatal leuprolide treatment (Figure S2G); other variables in PreN rats, such as glucose (Figure S2L), total cholesterol (Figure S2C), triglycerides (Figure S2K), and triglyceride-glucose (TyG) index (Figure S2O), did not modify after leuprolide treatment. PostN rats, in turn, did not show any metabolic modification after administration of leuprolide acetate (Figure S2B, F, H, J, and N).

Triglyceride levels were increased in the PostN group (mean \pm SD 88.8 \pm 9.3 ) compared to those in the PreN group (mean $\pm \mathrm{SD} 63.5 \pm 7.2$ ) (Student's $t$-test, $P=0.04$ ). Overall, no changes in the triglyceride levels were identified in the three different groups (Figure 3D), although a significant higher glucose was observed in both androgenized rodent models $(P=0.001)$ (Figure 3F). The product of TyG was increased in PostN rats in comparison with that in the two other groups $(P=0.02)$ (Figure 3E).

As shown in Figure S2M, control rats treated with leuprolide acetate (control L) exhibited an increase in the TyG index, suggesting a worsening of metabolic control (Figure S2D). No other changes regarding total cholesterol, HDL-C, or triglycerides were reported in control rats (Figure S2A, E, and I). 


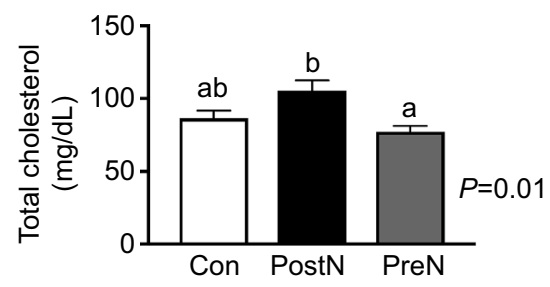

D

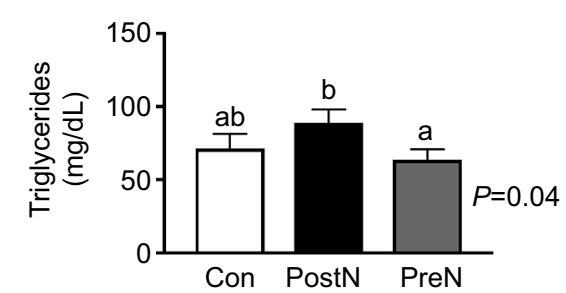

B

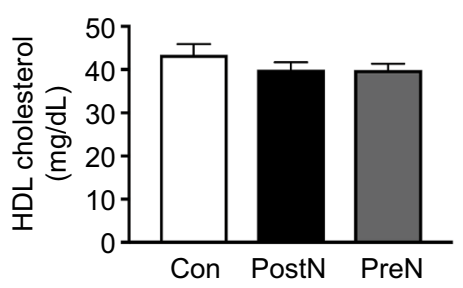

C

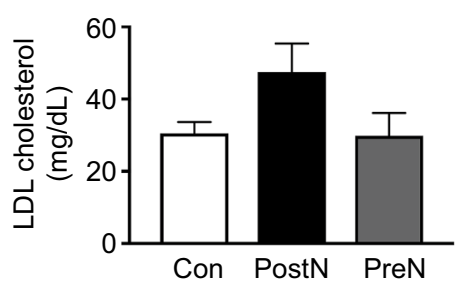

Figure 3 Serum levels of total cholesterol (A), HDL cholesterol (B), LDL cholesterol (C), triglycerides (D), TyG index (E), and glucose (F) in Con, PreN, and PostN androgenized rats.

Notes: Con $(n=8)$; PostN androgenized $(n=7)$; PreN androgenized $(n=8)$. (A) ANOVA $P=0.01$; (D) ANOVA $P=0.04 ;(E)$ ANOVA $P=0.02$; (F) ANOVA $P=0.00$ I. Distinct letters indicate a statistical significant difference (adjusted $P$ level $<0.05$ ) obtained with multi-comparison Tukey's test.

Abbreviations: ANOVA, analysis of variance; Con, controls; HDL, high-density lipoprotein; LDL, low-density lipoprotein; PostN, postnatal; PreN, prenatal; TyG, triglygeride-glucose.

Figure 4 summarizes the main dissimilarities between PreN and PostN androgenized rat protocols in our study.

\section{Discussion}

Women with PCOS most frequently exhibit dyslipidemia, glucose intolerance/diabetes mellitus, and increased oxidative stress marker levels. Our study evaluated whether different protocols of androgenization leading to normal and abnormal reproductive and metabolic rat phenotypes could be associated with a particular lipid status and redox balance in the blood. We show that a combination of changes in oxidative stress (increased direct oxidation and increased antioxidative profile) was observed in PostN rats exhibiting anovulation/ anestrous and increased TyG, whereas this phenomenon was not observed in ovulatory/estrous PreN rats.

All groups of rats achieved a similar final weight at the end of the study. As a result, it was possible to avoid possible bias related to higher adipose accumulation, which is a predominant characteristic of rodents following the use of implants with dihydrotestosterone (DHT), ${ }^{13,23}$ letrozole, ${ }^{23-26}$ or other miscellaneous protocols. ${ }^{27-29} \mathrm{We}$ also observed an earlier weight reduction in PreN group rats at birth and day 60. This finding was consistent with previous reports of rats androgenized with testosterone propionate prenatally.
An increase in direct oxidation, FRAP, was identified in the plasma of androgenized animals in our study. This result contrasts with the work of Daneasa et al (2016), ${ }^{30}$ where the serum levels of malondialdehyde (MDA), a lipid peroxidation marker, were similar between letrozole-treated rats and its controls. No modifications in MDA were also reported in the ovary tissue of Sprague-Dawley rats submitted to free testosterone ( 2 or $5 \mathrm{mg}$ ) administration, ${ }^{17}$ although an increase in lipid peroxidation products of the ovary was found in letrozole rats by another study. ${ }^{24}$

In the present study, antioxidant capacity was estimated in the serum of androgenized animals and controls through the levels of albumin. Albumin is capable of scavenging hydroxyl radicals with its reduced $(-\mathrm{SH})$ cysteine residue (Cys34) and, therefore, considered as one of the major antioxidant elements in the blood of humans and rats. ${ }^{31-36}$ We identified increased serum albumin levels in PostN rats compared with controls and the PreN group. In a previous study, no differences in glutathione peroxidase (GPx), another antioxidant marker, were described between letrozole rats and controls. ${ }^{30}$ However, an increase in the antioxidant capacity measured by catalase activity and superoxide dismutase or GPx has been identified in the ovary of PCOS rats..$^{17,24,30}$ 


\begin{tabular}{|c|c|c|}
\hline & $\begin{array}{l}\text { PostN group } \\
\text { - Anovulatory cycles (anestrous) } \\
\text { - Increased number atertic follicles } \\
\text { - Decreased corpus luteum }\end{array}$ & $\begin{array}{l}\text { PreN group } \\
\text { - Ovulatory cycles (estrous cycles) } \\
\text { - Ovary histology (number of atretic } \\
\text { and healthy follicles, corpus } \\
\text { luteum) similar to control rats }\end{array}$ \\
\hline $\begin{array}{l}\text { Oxidative stress markers } \\
\text { (FRAP, AOPP, TOS, \# Albumin) }\end{array}$ & $\begin{array}{l}\text { Increased (FRAP)* } \\
\text { Increased (Albumin)* }\end{array}$ & $\begin{array}{l}- \\
-\end{array}$ \\
\hline Glucose & Increased* & Increased $^{*}$ \\
\hline TyG Index & Increased* & - \\
\hline Total cholestrol & Increased & - \\
\hline HDL cholestrol & - & - \\
\hline Weight & $\begin{array}{c}-(d 1) \\
-(d 90) \\
-(d 110)\end{array}$ & $\begin{array}{l}\text { Decreased }(\mathrm{d} 1)^{*} \\
\text { Decreased }(\mathrm{d} 90) \\
-(\mathrm{d} 110)\end{array}$ \\
\hline
\end{tabular}

Figure 4 Metabolic and oxidative stress markers' characteristics of Post $\mathrm{N}$ and PreN rats.

Notes: *Significant differences with control rats. - indicates similarities with controls. The full reproductive aspects of these groups (PreN, PostN, and control with and without treatment with $\mathrm{GnRH}$ agonists) have been published in a previous study. ${ }^{19}$ Our results agreed with those of previous studies that employed testosterone propionate, ${ }^{12,18,44,45}$ with few exceptions. ${ }^{46}$

Abbreviations: AOPP, advanced oxidation protein product; FRAP, ferric-reducing ability of plasma; GnRH, gonadotropin-releasing hormone; HDL, high-density lipoprotein; PostN, postnatal; PreN, prenatal; TOS, total oxidation status; TyG, triglygeride-glucose.

One reason for the divergences among experimental studies may be based on the existence of several approaches for the development of animal models of PCOS. Because of the diversity and limitation of rodent models, it has been claimed that there is no "gold standard" reproducing all abnormalities seen in PCOS. ${ }^{37}$ For this reason, caution is necessary to avoid an indiscriminate generalization of the meaning of PreN and PostN models regarding the presented data.

Our results suggested a dual augmentation in oxidative and antioxidative statuses that agreed with some findings in the blood of women with PCOS. In a previous meta-analysis, the mean of MDA, a direct oxidant marker, was $\sim 40 \%$ higher in PCOS than in controls. ${ }^{38}$ Other direct oxidants such as dimethylarginine and homocysteine and nitric acid were also increased. However, in the same study, antioxidant markers were reduced (glutathione), increased (superoxide dismutase activity), or equal (total antioxidant capacity) to controls. ${ }^{38}$

A recent study (544 PCOS and 468 control women) showed that all four typical phenotypes of PCOS based on the Rotterdam criteria were associated with higher TOS and oxidative stress index (OSI) in comparison with control women. ${ }^{11}$ Increased oxidative stress in PCOS was related to higher plasma glucose and triglycerides. ${ }^{11}$ Remarkably, all oligo-anovulatory PCOS women, except PCOS women with presumed regular cycles, show an increased total antioxidant capacity in the serum. ${ }^{11}$ In our study, anovulatory rats (PostN) presented an increased TyG index. TyG, the product of triglycerides with glucose, has been considered in humans and in rodents as a surrogate marker of insulin resistance and metabolic syndrome. ${ }^{39-42}$ We showed that only PostN rats exhibited statistically significant higher TyG indices than controls, which may represent an additional link toward the disruption of the oxidative stress markers. ${ }^{43}$

Although the comparison of two models (PreN and PostN) with an extra ovulatory control (PostN L rats) consisted in one strength of the present study, weakness should be considered as well. The realization of euthanasia in rats at different estrous cycles may also had an impact of estrogens on oxidative stress markers. Other limitations of our research from our point of view were related to sample size, the absence of subgroups (lean, obese/young, aged), and the lack of inclusion of more antioxidant stress markers to the study.

\section{Conclusion}

The results presented suggest that an increased direct oxidation and an increased antioxidative capacity could be associated with PostN treatment with testosterone propionate (PostN), which is usually linked with anovulatory cycles and insulin resistance estimated by higher TyG. Androgenized rats treated with leuprolide acetate (PostN L) and presenting 
estrous cycles did not exhibit modifications in biochemical status or increased direct oxidation (FRAP) in plasma but showed a reduced antioxidant capacity estimated by albumin serum levels. PreN androgenization, in turn, was related to a lower weight at birth but a less harmful phenotype. Altogether, these findings continue to support the central role of androgen excess, anovulation, and insulin resistance as the key factors to trigger redox abnormalities in PCOS. Additional studies of the impact of weight gain, high-glucose/ high-fat diet, ${ }^{43}$ or simply the long-term follow-up will improve the comprehension of the intricacy mechanisms of oxidative stress in PCOS.

\section{Acknowledgment}

This work was supported by Capes Foundation and the National Council for Scientific and Technological Development (CNPq), Brazil, grant 445019/2014-0.

\section{Author contributions}

All authors made substantial contributions to conception and design, acquisition of data, or analysis and interpretation of data; took part in drafting the article or revising it critically for important intellectual content; gave final approval of the version to be published; and agree to be accountable for all aspects of the work.

\section{Disclosure}

The authors report no conflicts of interest in this work.

\section{References}

1. Franks S. Polycystic ovary syndrome. N Engl J Med. 1995;333(13): 853-861.

2. Norman RJ, Dewailly D, Legro RS, Hickey TE. Polycystic ovary syndrome. Lancet. 2007;370(9588):685-697.

3. Balen A, Homburg R, Franks S. Defining polycystic ovary syndrome. BMJ. 2009;338:a2968.

4. Diamanti-Kandarakis E, Argyrakopoulou G, Economou F, Kandaraki E, Koutsilieris M. Defects in insulin signaling pathways in ovarian steroidogenesis and other tissues in polycystic ovary syndrome (PCOS). J Steroid Biochem Mol Biol. 2008;109(3-5):242-246.

5. Deepika ML, Nalini S, Maruthi G, et al. Analysis of oxidative stress status through MN test and serum MDA levels in PCOS women. Pak J Biol Sci. 2014;17(4):574-577.

6. Azziz R, Carmina E, Chen Z, et al. Polycystic ovary syndrome. Nat Rev Dis Primers. 2016;2:16057.

7. Victor VM, Rovira-Llopis S, Banuls C, et al. Insulin resistance in PCOS patients enhances oxidative stress and leukocyte adhesion: role of myeloperoxidase. PLoS One. 2016;11(3):e0151960.

8. Abruzzese GA, Cerrrone GE, Gamez JM, et al. Lipid accumulation product (LAP) and Visceral Adiposity Index (VAI) as markers of insulin resistance and metabolic associated disturbances in young argentine women with polycystic ovary syndrome. Horm Metab Res. 2017;49(1):23-29.

9. Banuls C, Rovira-Llopis S, Martinez de Maranon A, et al. Metabolic syndrome enhances endoplasmic reticulum, oxidative stress and leukocyte-endothelium interactions in PCOS. Metabolism. 2017;71:153-162.
10. Behboudi-Gandevani S, Amiri M, Bidhendi Yarandi R, et al. The risk of metabolic syndrome in polycystic ovary syndrome: a systematic review and meta-analysis. Clin Endocrinol (Oxf). 2017;88(2):169-184.

11. Zhang R, Liu H, Bai H, et al. Oxidative stress status in Chinese women with different clinical phenotypes of polycystic ovary syndrome. Clin Endocrinol (Oxf). 2017;86(1):88-96.

12. Slob AK, den Hamer R, Woutersen PJ, van der Werff ten Bosch JJ. Prenatal testosterone propionate and postnatal ovarian activity in the rat. Acta Endocrinol (Copenh). 1983;103(3):420-427.

13. Manneras L, Cajander S, Holmang A, et al. A new rat model exhibiting both ovarian and metabolic characteristics of polycystic ovary syndrome. Endocrinology. 2007;148(8):3781-3791.

14. Motta AB. Dehydroepiandrosterone to induce murine models for the study of polycystic ovary syndrome. J Steroid Biochem Mol Biol. 2010;119(3-5):105-111.

15. Manneras-Holm L, Leonhardt H, Kullberg J, et al. Adipose tissue has aberrant morphology and function in PCOS: enlarged adipocytes and low serum adiponectin, but not circulating sex steroids, are strongly associated with insulin resistance. J Clin Endocrinol Metab. 2011;96(2):E304-E311.

16. Heber MF, Ferreira SR, Velez LM, Motta AB. Prenatal hyperandrogenism and lipid profile during different age stages: an experimental study. Fertil Steril. 2013;99(2):551-557.

17. Amalfi S, Velez LM, Heber MF, et al. Prenatal hyperandrogenization induces metabolic and endocrine alterations which depend on the levels of testosterone exposure. PLoS One. 2012;7(5):e37658.

18. Tyndall V, Broyde M, Sharpe R, Welsh M, Drake AJ, McNeilly AS. Effect of androgen treatment during foetal and/or neonatal life on ovarian function in prepubertal and adult rats. Reproduction. 2012;143(1):21-33.

19. Serrano Mujica LK, Bertolin K, Bridi A, et al. The impact of postnatal leuprolide acetate treatment on reproductive characteristics in a rodent model of polycystic ovary syndrome. Mol Cell Endocrinol. 2017;442:125-133.

20. Benzie IF, Strain JJ. The ferric reducing ability of plasma (FRAP) as a measure of "antioxidant power": the FRAP assay. Anal Biochem. 1996;239(1):70-76.

21. Medeiros MS, Schumacher-Schuh A, Cardoso AM, et al. Iron and oxidative stress in Parkinson's disease: an observational study of injury biomarkers. PLoS One. 2016;11(1):e0146129.

22. Erel O. A new automated colorimetric method for measuring total oxidant status. Clin Biochem. 2005;38(12):1103-1111.

23. Hoang V, Bi J, Mohankumar SM, Vyas AK. Liraglutide improves hypertension and metabolic perturbation in a rat model of polycystic ovarian syndrome. PLoS One. 2015;10(5):e0126119.

24. Pandey V, Singh A, Singh A, Krishna A, Pandey U, Tripathi YB. Role of oxidative stress and low-grade inflammation in letrozole-induced polycystic ovary syndrome in the rat. Reprod Biol. 2016;16(1):70-77.

25. Maliqueo M, Sun M, Johansson J, et al. Continuous administration of a P450 aromatase inhibitor induces polycystic ovary syndrome with a metabolic and endocrine phenotype in female rats at adult age. Endocrinology. 2013;154(1):434-445.

26. Ullah A, Jahan S, Razak S, et al. Protective effects of GABA against metabolic and reproductive disturbances in letrozole induced polycystic ovarian syndrome in rats. J Ovarian Res. 2017;10(1):62.

27. Walters KA, Allan CM, Handelsman DJ. Rodent models for human polycystic ovary syndrome. Biol Reprod. 2012;86(5):112-141.

28. Wu C, Lin F, Qiu S, Jiang Z. The characterization of obese polycystic ovary syndrome rat model suitable for exercise intervention. PLoS One. 2014;9(6):e99155.

29. Marcondes RR, Carvalho KC, Duarte DC, et al. Differences in neonatal exposure to estradiol or testosterone on ovarian function and hormonal levels. Gen Comp Endocrinol. 2015;212:28-33.

30. Daneasa A, Cucolas C, Lenghel LM, Olteanu D, Orasan R, Filip GA. Letrozole vs estradiol valerate induced PCOS in rats: glycemic, oxidative and inflammatory status assessment. Reproduction. 2016;151(4): 401-409.

31. Sitar ME, Aydin S, Cakatay U. Human serum albumin and its relation with oxidative stress. Clin Lab. 2013;59(9-10):945-952. 
32. Ciapetti M, Mancinelli P, Cecchi A, Borrelli E, Bocci V, Peris A. Reduction of non-enzymatic antioxidants in plasma during ECMO-treatment in ARDS by influence A H1N1. J Crit Care. 2017;43:220-224.

33. Kinoshita $H$, Watanabe $\mathrm{K}$, Azma $\mathrm{T}$, et al. Human serum albumin and oxidative stress in preeclamptic women and the mechanism of albumin for stress reduction. Heliyon. 2017;3(8):e00369.

34. Mandic A, Cavar I, Skoro I, et al. Body composition and inflammation in hemodialysis patients. Ther Apher Dial. 2017;21(6):556-564.

35. Masudo R, Yasukawa K, Nojiri T, et al. Evaluation of human nonmercaptalbumin as a marker for oxidative stress and its association with various parameters in blood. J Clin Biochem Nutr. 2017;61(2):79-84.

36. Prieto AKC, Gomes-Filho JE, Azuma MM, et al. Influence of apical periodontitis on stress oxidative parameters in diabetic rats. J Endod. 2017;43(10):1651-1656.

37. Maliqueo M, Benrick A, Stener-Victorin E. Rodent models of polycystic ovary syndrome: phenotypic presentation, pathophysiology, and the effects of different interventions. Semin Reprod Med. 2014;32(3):183-193.

38. Murri M, Luque-Ramirez M, Insenser M, Ojeda-Ojeda M, EscobarMorreale HF. Circulating markers of oxidative stress and polycystic ovary syndrome (PCOS): a systematic review and meta-analysis. Hum Reprod Update. 2013;19(3):268-288.

39. Simental-Mendia LE, Rodriguez-Moran M, Guerrero-Romero F. The product of fasting glucose and triglycerides as surrogate for identifying insulin resistance in apparently healthy subjects. Metab Syndr Relat Disord. 2008;6(4):299-304.
40. Gonzalez-Torres L, Vazquez-Velasco M, Olivero-David R, et al. Glucomannan and glucomannan plus spirulina added to pork significantly block dietary cholesterol effects on lipoproteinemia, arylesterase activity, and CYP7A1 expression in Zucker fa/fa rats. J Physiol Biochem. 2015;71(4):773-784.

41. Pinto BA, Melo TM, Flister KF, et al. Early and sustained exposure to high-sucrose diet triggers hippocampal ER stress in young rats. Metab Brain Dis. 2016;31(4):917-927.

42. Qu C, Zhou X, Yang G, Li L, Liu H, Liang Z. The natural logarithm of zinc-alpha2-glycoprotein/HOMA-IR is a better predictor of insulin sensitivity than the product of triglycerides and glucose and the other lipid ratios. Cytokine. 2016;79:96-102.

43. Szczuko M, Zapalowska-Chwyc M, Maciejewska D, Drozd A, Starczewski A, Stachowska E. High glycemic index diet in PCOS patients. The analysis of IGF I and TNF-alpha pathways in metabolic disorders. Med Hypotheses. 2016;96:42-47.

44. Fels E, Bosch LR. Effect of prenatal administration of testosterone on ovarian function in rats. Am J Obstet Gynecol. 1971;111(7): 964-969.

45. Huffman L, Hendricks SE. Prenatally injected testosterone propionate and sexual behavior of female rats. Physiol Behav. 1981;26(5): 773-778.

46. Swanson HE, Werff ten Bosch JJ. The "early-androgen" syndrome; effects of pre-natal testosterone propionate. Acta Endocrinol (Copenh). 1965;50(3):379-390. 


\section{Supplementary materials}

A

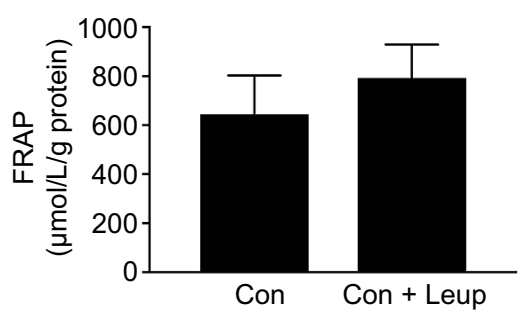

D

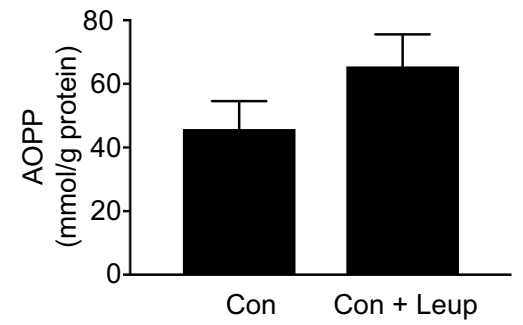

G

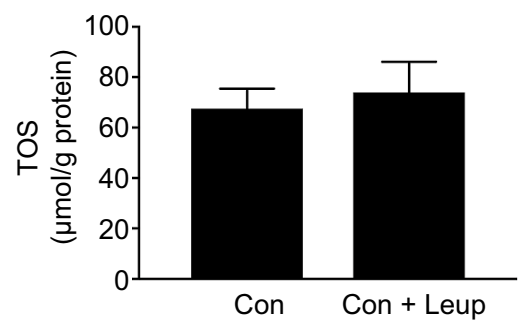

J

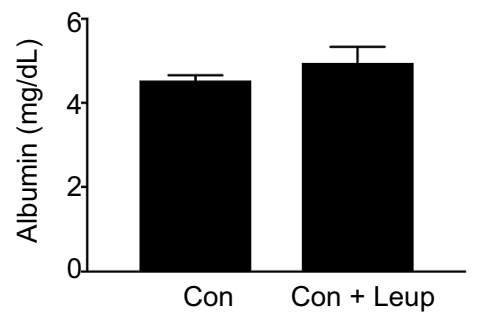

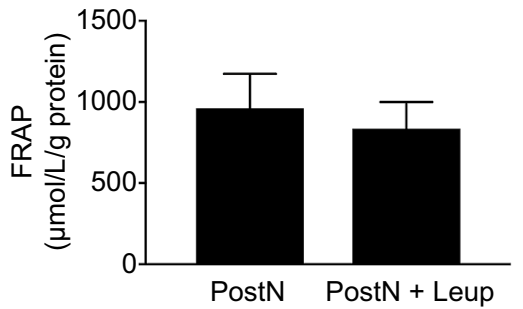

E

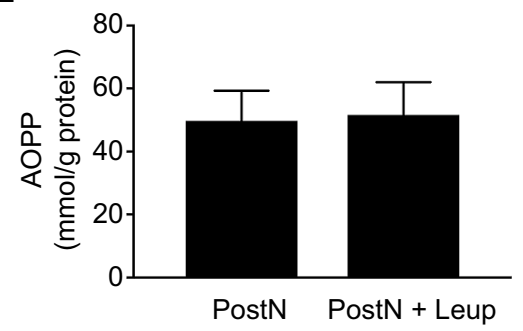

H

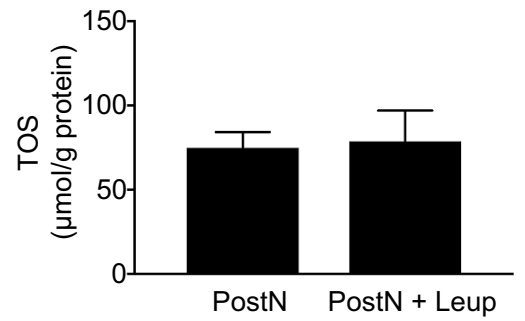

K

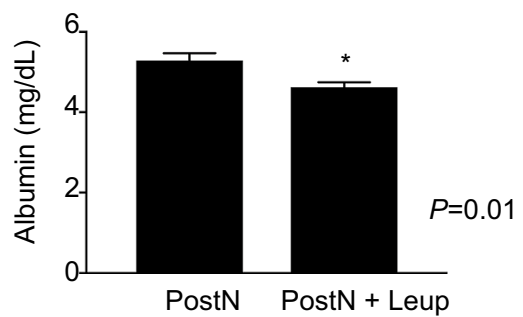

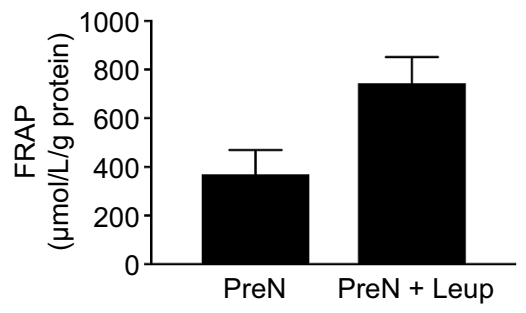

$\mathbf{F}$
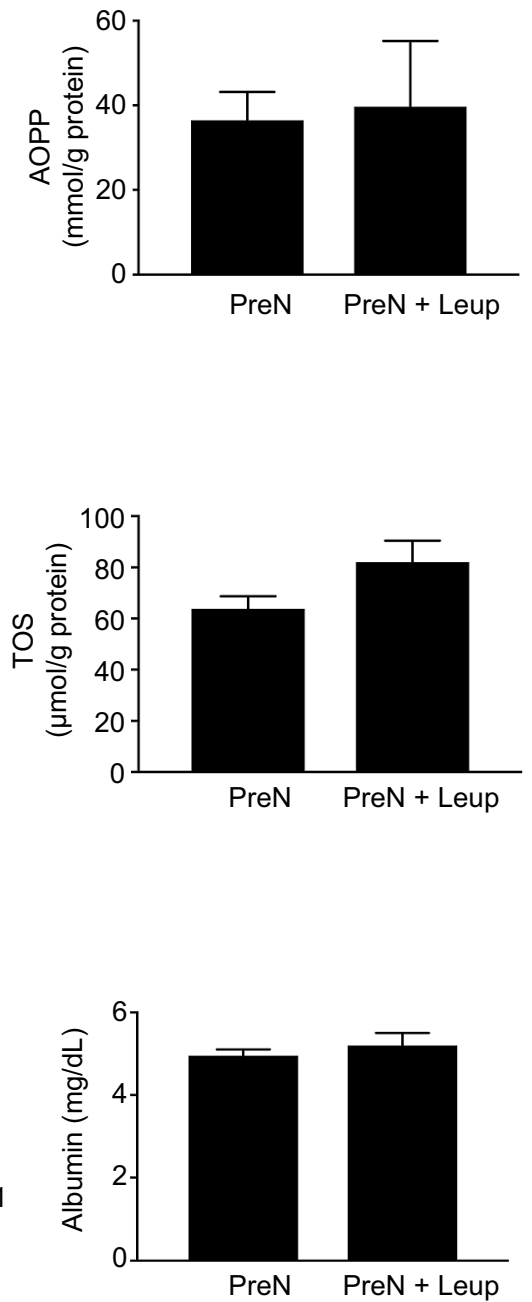

Figure SI Oxidative stress markers in $\mathrm{C}, \mathrm{PreN}$, and PostN androgenized rats, which were treated and not treated with neonatal leuprolide acetate.

Notes: (A-C) FRAP; (D-F) AOPP; (G-I) TOS; (J-L) Albumin. Con ( $n=8)$; Con + Leup, Con treated with leuprolide; PostN androgenized ( $n=7$ ); PostN + Leup, PostN androgenized treated with leuprolide ( $n=7)$; PreN androgenized $(n=8)$; PreN + Leup, PreN androgenized treated with leuprolide ( $n=4)$. Results were reported as mean (SEM). Statistical analysis used Student's t-test. *Significance was assumed at $P<0.05$.

Abbreviations: AOPP, advanced oxidation protein product; Con, controls; FRAP, ferric-reducing ability of plasma; Leup, leuprolide; PostN, postnatal; PreN, prenatal; SEM, standard error of the mean; TOS, total oxidation status. 


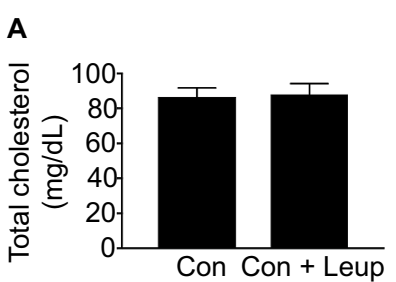

E

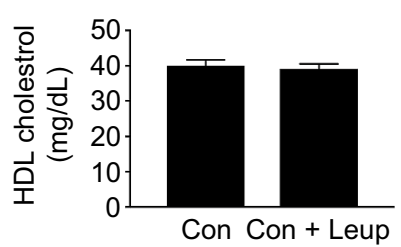

I

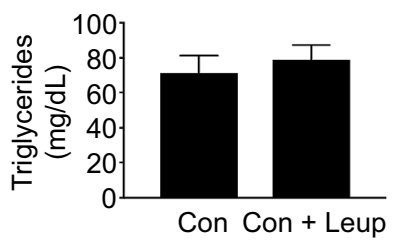

M

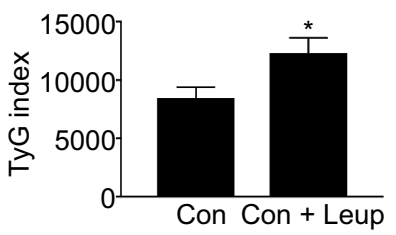

B

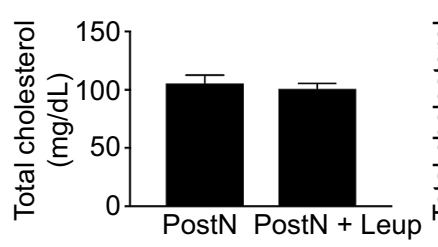

F

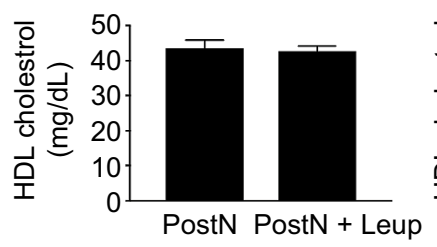

C

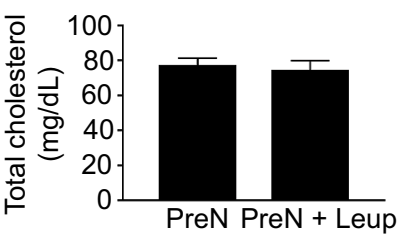

D

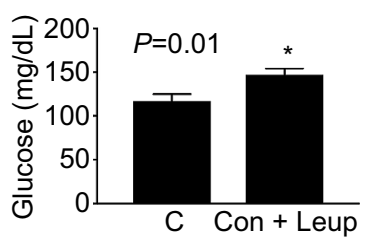

G

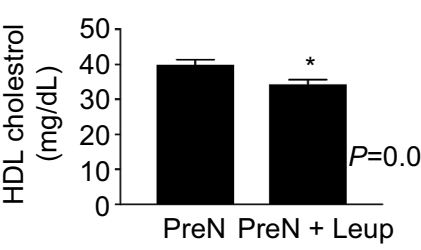

H

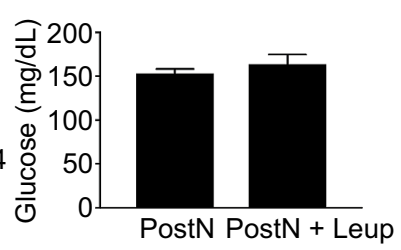

$\mathbf{J}$
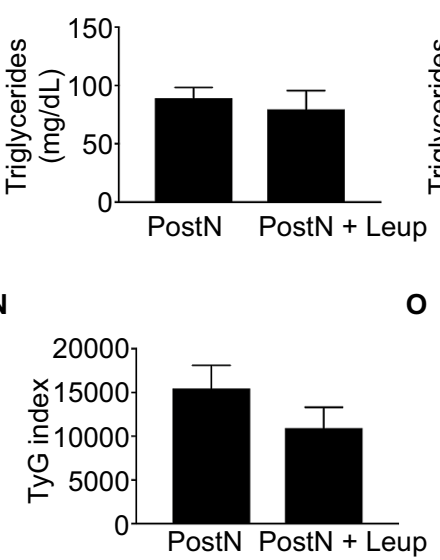

K

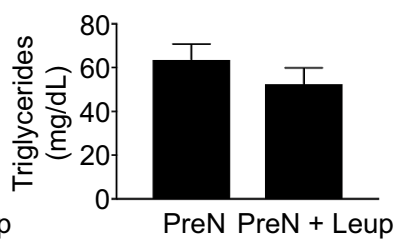

L

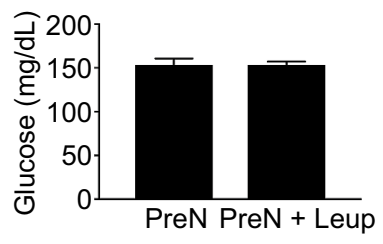

Figure S2 Biochemical markers.

Notes: Serum levels of total cholesterol (A-C), HDL cholesterol (E-G), triglycerides (I-K), TyG index (M-O), and glucose (D, H, and $\mathbf{L})$ in Con, PreN, and PostN rats submitted or not submitted to the neonatal treatment with leuprolide acetate. Con $(n=8) ; C+$ Leup, Con treated with leuprolide ( $n=6)$; Post $N$ androgenized ( $n=7)$; Post $N+$ Leup, Post $N$ androgenized treated with leuprolide $(n=7)$; PreN androgenized $(n=8)$; PreN + Leup, PreN androgenized treated with leuprolide ( $n=4)$. Results were reported as mean (SEM). Statistical analysis used Student's t-test. *Significance was assumed at $P<0.05$.

Abbreviations: Con, controls; HDL, high-density lipoprotein; Leup, Leuprolide; PostN, postnatal; PreN, prenatal; SEM, standard error of the mean; TyG, triglygerideglucose.

\section{Publish your work in this journal}

The Journal of Inflammation Research is an international, peer-reviewed open access journal that welcomes laboratory and clinical findings on the molecular basis, cell biology and pharmacology of inflammation including original research, reviews, symposium reports, hypothesis formation and commentaries on: acute/chronic inflammation; mediators of

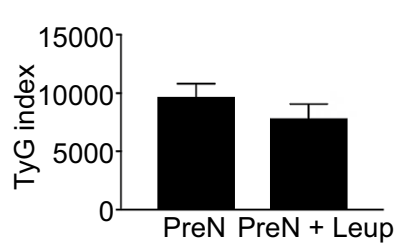

inflammation; cellular processes; molecular mechanisms; pharmacology and novel anti-inflammatory drugs; clinical conditions involving inflammation. The manuscript management system is completely online and includes a very quick and fair peer-review system. Visit http://www.dove press.com/testimonials.php to read real quotes from published authors. 